\title{
Síndrome de burnout en profesionales de la salud: una revisión sistemática durante la COVID-19
}

Burnout syndrome in health professionals in times of COVID-19: a systematic review

Síndrome de Burnout em profissionais de saúde: uma revisão sistemática durante a COVID-19

Calixto Tapullima-Mori

calixtotapullima01@gmail.com CNL ASESORES-Perú

https://orcid.org/0000-0001-8036-2199

Gustavo Ramírez García

gustavoramirezgarcia2@gmail.com

Universidad César Vallejo-Perú https://orcid.org/0000-0003-0035-7088

Janina Saavedra Meléndez

psicologasaavedra@hotmail.com Universidad César Vallejo https://orcid.org/0000-0002-7571-7271

\section{RESUMEN}

La COVID-19 se propagó muy rápido a nivel mundial, si bien la población en general se ha visto afectada, los colectivos de mayor nivel de vulnerabilidad al virus y sus consecuencias de tipo psicológico son el personal de salud. Se planteó como objetivo evaluar el síndrome de burnout en profesionales de la salud en tiempos de la COVID-19 en bases de datos que contengan literatura científica. Se trabajaron bajo un enfoque cualitativo y se analizó 19 artículos de fuentes primarias indexados en Scielo, Redalyc, Scopus, DOAJ, Dialnet y Ebsco. En primera instancia se obtuvo 1586 artículos, de éstos solo 19 cumplieron los criterios planteados. Se observó una mayor presencia de artículos con diseño descriptivo observacional transversales, la muestra total fue profesionales de la salud, donde los índices altos de burnout llegaron al 95\% de la totalidad de los artículos analizados y con mayor prevalencia en mujeres que en hombres, concluyendo así que el burnout es alto en los profesionales de la salud; además, para la presencia de burnout, el contacto con pacientes COVID-19, los horarios y la sobrecarga laboral han sido un factor primordial para que se desencadene su sintomatología.

Palabras clave / Descriptores: burnout; COVID-19; personal de salud; agotamiento emocional; despersonalización

\begin{abstract}
COVID-19 spread very quickly worldwide, although the general population has been affected, the groups with the highest level of vulnerability to the virus and its psychological consequences are health personnel. The objective was to evaluate burnout syndrome in health professionals in times of COVID-19 in databases containing scientific literature. We worked under a qualitative approach and analyzed 19 articles from primary sources indexed in Scielo, Redalyc, Scopus, DOAJ, Dialnet and Ebsco. In the first instance, 1586 articles were obtained, of which only 19 met the criteria set. A greater presence of articles with cross-sectional observational descriptive design was observed, the total sample was health professionals, where high burnout rates reached $95 \%$ of all the articles analyzed and with a higher prevalence in women than in men, thus concluding that burnout is high in health professionals; In addition, for the presence of burnout, contact with COVID-19 patients, schedules and work overload have been a primary factor for their symptoms to be triggered.
\end{abstract}

Keywords: burnout; COVID-19; health personnel; emotional exhaustion; depersonalization.

\section{RESUMO}

O COVID-19 se espalhou muito rapidamente pelo mundo, embora a população em geral tenha sido afetada, os grupos com maior nível de vulnerabilidade ao vírus e suas consequências psicológicas são o pessoal de saúde. O objetivo foi avaliar a síndrome de burnout em profissionais de saúde em tempos de COVID-19 em bases de dados contendo literatura científica. Trabalhamos sob abordagem qualitativa e analisamos 19 artigos de fontes primárias indexadas em Scielo, Redalyc, Scopus, DOAJ, Dialnet e Ebsco. Em primeira instância, foram obtidos 1.586 artigos, dos quais apenas 19 atenderam aos critérios estabelecidos. Observou-se maior presença de artigos com desenho descritivo observacional 
transversal, a amostra total foi de profissionais de saúde, onde as altas taxas de burnout atingiram $95 \%$ de todos os artigos analisados e com maior prevalência em mulheres do que em homens, concluindo assim que o burnout é elevado nos profissionais de saúde; Além disso, para a presença de burnout, o contato com pacientes COVID-19, horários e sobrecarga de trabalho têm sido um fator primordial para que seus sintomas sejam acionados.

Palavras-chave: burnout; COVID-19; pessoal de saúde; exaustão emocional; despersonalização

\section{INTRODUCCIÓN}

La COVID-19 se registró a finales del 2019, repercutiendo de esta manera en una pandemia mundial de muy rápida propagación. Si bien la población en general se ha visto afectada, los colectivos de mayor nivel de vulnerabilidad al virus y sus consecuencias de tipo psicológico fueron los profesionales de salud (Rodríguez \& Sánchez, 2020); estos no solo están afectos al problema de salud mental que causa su constante involucramiento, sino, además, dificulta la capacidad de respuesta y toma de decisiones en las complicaciones urgentes en el desarrollo de sus actividades (Ricci-Cabello et al., 2020); siendo los periodos prolongados de confinamiento dentro de los nosocomios, la sobre carga y el aumento de los niveles de ansiedad que ha acarreado la angustia o agotamiento (Visser, 2020; Yakut et al., 2020). El burnout puede estar presente en cualquiera de las áreas, más aún con la presencia de factores externos como la atención a pacientes con diagnóstico positivo de COVID-19 o aquellos que se encuentran a la espera de confirmación (Seow et al., 2020). De acuerdo con diversos estudios se manifiesta que se han observado elevados niveles de agotamiento en personal médico de atención a pacientes COVID-19 afectando no solo su bienestar físico y psicológico, sino además su calidad de vida (Çelmeçe \& Menekay, 2020). Por su parte Burki (2020) manifiesta que de 1520 sujetos de más de 101 países, se registró agotamiento en $38 \%$ a consecuencia de la primeras apariciones de la COVID-19; de esta manera resulta prudente enfatizar sobre la importancia de los factores de riesgo que afectan al personal médico y plantear estrategias de prevención (El-Hage et al., 2020). De acuerdo con la realidad que se ha mencionado, la prevalencia del agotamiento, síndrome de burnout, desgaste u angustia está presente en el personal médico que atiende a pacientes COVID-19, razón por la cual un estudio de carácter sistémico contribuye no solo a conocer su presencia en los profesionales de salud, sino, además, a la recopilación documentaria impulsada por la realidad actual de crisis sanitaria. En ese sentido el estudio busca evaluar el síndrome de burnout en profesionales de la salud en tiempo de COVID-19 en base de datos que contengan artículos científicos de fuentes primarias.

\section{FUNDAMENTO TEÓRICO}

\subsection{Antecedentes de revisión}

De acuerdo con un análisis de la bibliografía, se ha logrado identificar diversos estudios que hacen énfasis en la pandemia y sus afecciones en diversos aspectos de la salud mental, como el caso de Stuijfzand et al. (2020) quienes plantearon como objetivo una revisión rápida sobre evidencias de impacto psicológico de las pandemias en la salud mental de los profesionales de salud, para ello buscaron en revistas como embase.com, PubMed, APA Psycinfo-Ovid SP y Web of Science, incluyeron artículos escritos en francés o inglés. De 1308 artículos sólo 50 fueron incluidos por cumplir con los datos establecidos, llegaron a concluir que el personal médico que laboran en atención a pacientes durante la epidemia/pandemia presentan una mayor predisposición a padecer problemas de salud mental a corto y largo plazo, de igual manera estos pueden incidir en su calidad de vida. Finalmente, resulta necesario que se establezcan programas de prevención temprana por cuanto el apoyo psicosocial contribuirá en su bienestar y en la calidad de atención al paciente; Sharifi et al. (2020) plantearon efectuar una revisión sistemática de la epidemiología del agotamiento y las estrategias y recomendaciones para la prevención o reducción en los colaboradores médicos de las salas COVID-19, se buscó información en las bases de datos de Medline, Science Direct y Scopus, se incluyeron 12 estudios, donde concluyeron que para hacer 
frente a los problemas de salud mental en el personal con atención en salas COVID-19 es necesario el ajuste de los turnos de trabajo, la reducción de los factores de estrés que se relacionan con el trabajo, creando un ambiente laboral saludable previniendo así el burnout. Por su parte Sanghera et al. (2020) plantearon en su estudio determinar el impacto del SARS-CoV-2 en la salud mental de los colaboradores sanitarios, la búsqueda se efectúo en las bases de datos OVid Medline y Embase, incluyendo así 44 artículos, llegando a concluir que el COVID-19 ha tenido una afección significativa en la salud mental de los colaboradores, siendo los de mayor perjuicio aquellos que trabajan en primera línea de atención; de igual manera Amanullah \& Ramesh (2020) planteó como objetivo efectuar un análisis y resumen actual sobre factores que contribuyen en el desgaste profesional (burnout) en medio de la pandemia, buscaron bibliografía en la base de datos de Medline y Embase, incluyendo 5 artículos científicos, presentando como conclusión que pese al número reducido de documentos la atención se estimó que si bien la COVID-19 produjo nuevos desafíos en el trabajo para los profesionales de la salud, estos no se relacionan estrechamente con el incremento de un mayor nivel de desgaste, además la atención plena podría reducir la aparición del burnout y sus componentes.

\subsection{Definiciones conceptuales}

Se ha explorado diversas teorías de burnout como los modelos presentados por Reed (1981); que definen como el proceso de desencanto al trabajo o las actividades, siendo sus fases el entusiasmo, estancamiento y frustración; por su parte Cherniss (1993) define al burnout como la combinación del estrés y tensión que se vive en el centro de trabajo, presentando así, tres momentos en las que se desencadenan; el primero se evidencia en el desequilibrio que se registra entre las demandas en el trabajo y los recursos individuales con la que cuenta la persona (estrés), el segundo hace alusión a la respuesta emocional en el momento que surge por el desequilibrio inicial, que se caracteriza por la ansiedad, tensión, fatiga y agotamiento (Carlin \& Garcés De Los Fayos, 2010) y el tercero plantea el cambio que se registra de las actitudes y conductas (afrontamiento defensivo). Sin embargo, el de mayor uso o frecuencia es el modelo teórico tridimensional, en las que se hace análisis de los componentes como el agotamiento emocional, despersonalización y la realización en el centro laboral, en ese sentido Maslach et al. (2001) manifiestan que el síndrome de burnout es una respuesta del individuo al estrés crónico que ha sido sometido a través del tiempo; desarrollando actitudes o comportamientos negativos que afectan su bienestar y el desarrollo de sus actividades, de esta manera la teoría plantea tres elementos como: agotamiento emocional, despersonalización y realización personal. De esta manera el análisis del SB en tiempos de COVID19 se ha convertido un reto para la salud pública como para la salud mental, además de considerar al grupo de mayor afección los profesionales de la salud (Juárez-García, 2020).

\section{PROCEDIMIENTOS METODOLÓGICOS}

\subsection{Enfoque, tipo y diseño}

El estudio ha comprendido un enfoque cualitativo, de tipo básico por cuanto la recopilación de los artículos permitieron sustentar la variable en estudio junto con la descripción de sus dimensiones, además, fueron investigaciones de recolección primaria (Montero \& León, 2002; Veléz et al., 2013). El diseño presentado fue teórico, de revisión sistemática, debido al abordaje de la variable Burnout (Ato et al., 2013), siendo así analizados e interpretados en el momento actual. 


\subsection{Población, muestra, muestreo}

Para el presente estudio se ha considerado como población al conjunto de revisiones sistemáticas efectuados en los últimos años que involucren la COVID-19 y el síndrome de burnout en profesionales de la salud, donde se exploró características, factores y magnitudes de su presencia. En cuanto a la muestra como parte representativa del conjunto de datos que se revisó (Otzen \& Manterola, 2017), se estableció 19 artículos que cumplieron con los criterios establecidos por el investigador, además el muestreo fue intencional por cuanto la población fue determinante en la selección de artículos, específicamente en aquellos que centren su atención en la COVID-19 y el síndrome de burnout. Los principales criterios de inclusión hacen mención de que los artículos informen sobre los índices de Burnout relacionados con la COVID-19 junto con artículos descriptivos, observacionales que cuenten con instrumentos de medición válidos. En cuanto a los criterios de exclusión se plantearon estudios de casos, revisiones, cartas al editor, estudios cualitativos y artículos incompletos.

\subsection{Técnicas e instrumentos}

Se revisó las bases de datos indexadas como Scielo, DOAJ, Dialnet, Redalyc, Ebsco y Scopus, haciendo uso de palabras clave como "síndrome de burnout" "Covid-19, burnout", "Sars cov", "pandemia" "agotamiento emocional", todo ello con la intención de obtener un amplio número de literatura científica (artículos) relevante para la investigación. Para el procesamiento de la información se consideraron estudios de acceso abierto, es importante mencionar que el estudio comprendió el año 2020 a febrero del 2021. Los datos obtenidos inicialmente fueron presentados en una matriz de sistematización, en la que se efectuó la depuración de información bajo los criterios de inclusión y exclusión establecidos, siendo estos procesados en el programa Excel, luego fueron presentados en los resultados a modo resumen. Se diseñó una matriz de registro de acuerdo con la base de datos extraídas, su sistematización estuvo determinada por elementos como el año de publicación, autor, el objetivo, muestra o participantes, instrumentos y conclusiones principales, luego de estas precisiones se consideraron separan en tres elementos correspondientes a las dimensiones para su descripción posterior.

\section{RESULTADOS Y DISCUSIÓN}

\subsection{Resultados}

Los datos fueron extraídos en primera instancia bajo el siguiente formato en la que se expresó la búsqueda de información. 


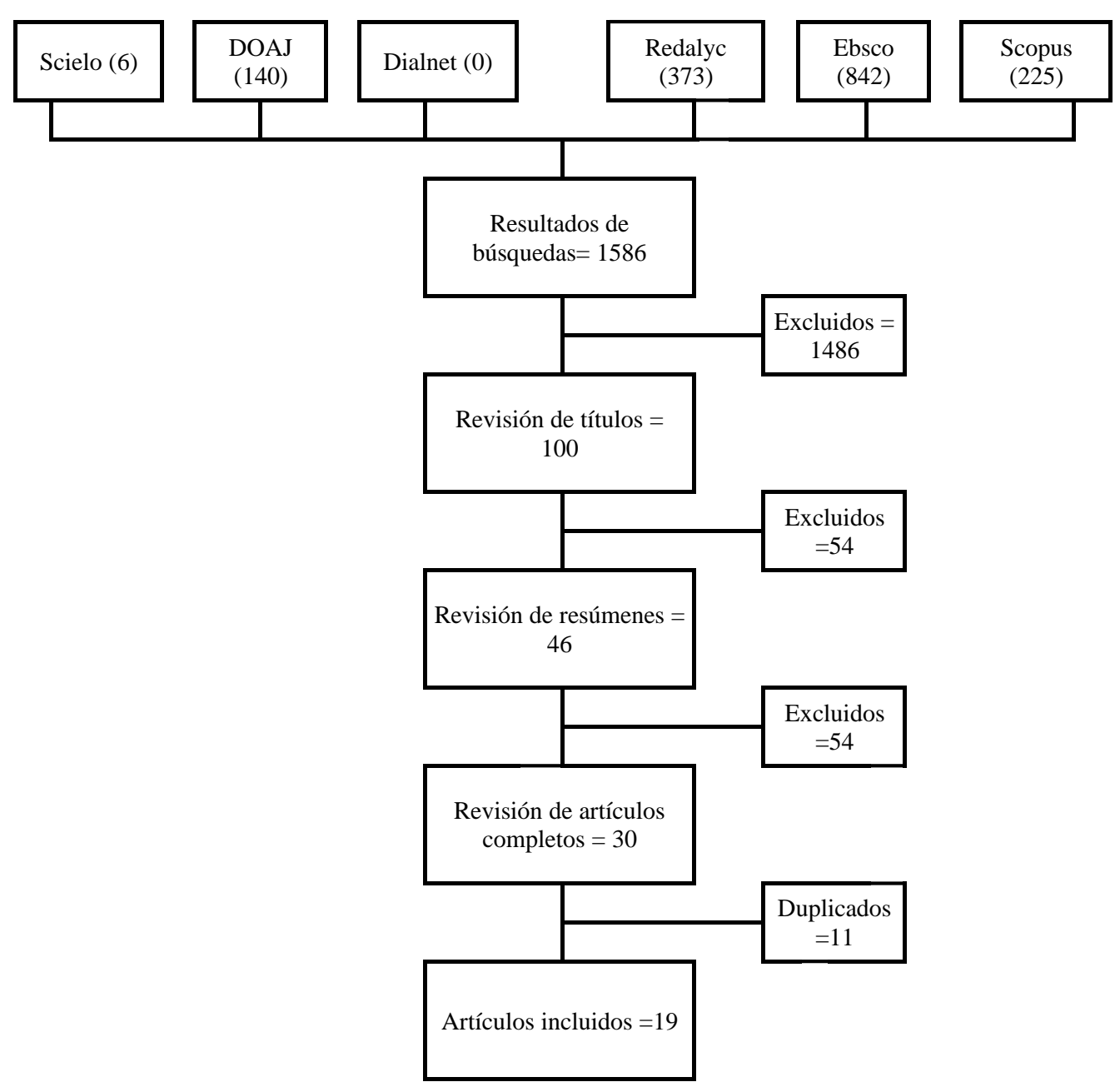

Figura 1 Flujo de información a través de las diferentes fases de revisión sistemática

Nota: Revisión bibliográfica

De acuerdo con el flujo de procesos de cuatro fases planteadas por Moher et al. (2009) en el proceso de selección de artículos científicos sobre el tema en mención se indagaron en seis bases de datos, obteniendo así una búsqueda inicial compuesta por 1586 artículos. Luego de la revisión de títulos, resúmenes, artículos completos y duplicidad se obtuvo solo 19 artículos para el desarrollo de la revisión. 
Tabla 1

Sistematización de los artículos

\begin{tabular}{|c|c|c|c|c|c|c|}
\hline $\mathbf{N}^{\circ}$ & Autores y año & País & Muestra & $\begin{array}{l}\text { Instrumento } \\
\text { principal }\end{array}$ & Objetivo general & Conclusiones \\
\hline 1 & $\begin{array}{l}\text { Vinueza et al. } \\
(2020)\end{array}$ & Ecuador & $\begin{array}{l}224 \text { médicos y } \\
\text { enfermeros }\end{array}$ & $\begin{array}{ll}\text { Inventario } & \text { de } \\
\text { Burnout } & \text { de } \\
\text { Maslach } & \end{array}$ & $\begin{array}{lr}\text { Determinar } & \text { la } \\
\text { presencia } & \text { e } \\
\text { intensidad del } & \text { SB } \\
\text { en médicos } & \text { y } \\
\text { enfermeros del } \\
\text { Ecuador durante la } \\
\text { pandemia. }\end{array}$ & $\begin{array}{l}\text { Durante la pandemia de la } \\
\text { covid19 el personal médico } \\
\text { ecuatoriano estuvo } \\
\text { expuesto a altos niveles de } \\
\text { burnout ( } 95 \%) \text {, presentes } \\
\text { con mayor frecuencia en } \\
\text { personal médico que en } \\
\text { enfermería sobre todo } \\
\text { jóvenes y mujeres. }\end{array}$ \\
\hline 2 & $\begin{array}{l}\text { Hoseinabadi et } \\
\text { al. (2020) }\end{array}$ & Irán & $\begin{array}{l}245 \\
\text { enfermeras }\end{array}$ & $\begin{array}{ll}\text { Inventario } & \text { de } \\
\text { Burnout } & \text { de } \\
\text { Oldenburg } & \\
\text { (OLBI); } & \\
\text { Cuestionario } \\
\text { de restrés } \\
\text { laboral (JSQ) }\end{array}$ & $\begin{array}{l}\text { Evaluar los niveles } \\
\text { de agotamiento } \\
\text { durante el brote de } \\
\text { covid19 e } \\
\text { identificar sus } \\
\text { principales factores } \\
\text { que influyen en las } \\
\text { enfermeras de } \\
\text { primera línea y } \\
\text { enfermeras de otras } \\
\text { salas. } \\
\end{array}$ & $\begin{array}{l}\text { Se identificó que el } \\
\text { agotamiento de las } \\
\text { enfermeras de primera } \\
\text { línea alcanzó un nivel } \\
\text { superior al de otras } \\
\text { enfermeras, siendo el estrés } \\
\text { laboral el factor } \\
\text { predominante para su } \\
\text { presencia. }\end{array}$ \\
\hline 3 & $\begin{array}{l}\text { De Moura et al. } \\
(2020)\end{array}$ & Brasil & 2708 médicos & $\begin{array}{l}\text { Oldenburg } \\
\text { Burnout } \\
\text { Inventory } \\
\text { (OLBI) }\end{array}$ & $\begin{array}{l}\text { Existe relación } \\
\text { entre la alta calidad } \\
\text { de supervisores y } \\
\text { médicos con la } \\
\text { reducción del nivel } \\
\text { de desgaste }\end{array}$ & $\begin{array}{l}\text { Es necesario desarrollar } \\
\text { relaciones de alto nivel } \\
\text { entre los supervisores y } \\
\text { médicos en general a fin de } \\
\text { evitar la propagación o } \\
\text { incremento de agotamiento, } \\
\text { sin embargo, queda } \\
\text { demostrado también que } \\
\text { los comportamientos de } \\
\text { liderazgo dentro del } \\
\text { escenario como la } \\
\text { pandemia es restringido y } \\
\text { quitado de sus aspectos } \\
\text { beneficiosos. }\end{array}$ \\
\hline 4 & $\begin{array}{l}\text { Duarte et al. } \\
(2020)\end{array}$ & Portugal & 2008 sujetos & $\begin{array}{l}\text { Copenhagen } \\
\text { Burnout } \\
\text { Inventory } \\
(\mathrm{CBI})\end{array}$ & $\begin{array}{l}\text { Evaluar al personal } \\
\text { sanitario rio el } \\
\text { síndrome de } \\
\text { burnout y factores } \\
\text { psicosociales. }\end{array}$ & $\begin{array}{l}\text { De manera general se ha } \\
\text { evidenciado un nivel } \\
\text { promedio de burnout en los } \\
\text { colaboradores de salud que } \\
\text { estos a su vez se dividieron } \\
\text { en dos grandes grupos de } \\
\text { agotamiento, en relación } \\
\text { con el agotamiento } \\
\text { personal fue alto en } 52.3 \% \\
\text { de participantes, así } \\
\text { también el agotamiento } \\
\text { asociado al trabajo fue alto } \\
\text { en el 53.1\% de los } \\
\text { encuestados. Cabe precisar } \\
\text { que el personal sanitario } \\
\text { que estuvo en contacto } \\
\text { directo con los pacientes } \\
\text { Covid alcanzaron un mayor } \\
\text { nivel de desgaste personal } \\
\text { y laboral. }\end{array}$ \\
\hline
\end{tabular}




\begin{tabular}{|c|c|c|c|c|c|c|}
\hline 5 & $\begin{array}{l}\text { Elhadi et al. } \\
(2020)\end{array}$ & Libia & $\begin{array}{l}532 \\
\text { trabajadores } \\
\text { de salud }\end{array}$ & $\begin{array}{ll}\text { Inventario } & \text { de } \\
\text { Burnout } & \text { de } \\
\text { Maslach } & \\
\text { abreviado } & \\
\text { (AMBI) } & \end{array}$ & $\begin{array}{l}\text { Determinar la } \\
\text { prevalencia de } \\
\text { burnout entre los } \\
\text { colaboradores } \\
\text { líbanos durante la } \\
\text { pandemia. }\end{array}$ & $\begin{array}{l}\text { De acuerdo con los } \\
\text { componentes de burnout, el } \\
67.1 \% \text { arrojaron } \\
\text { agotamiento emocional } \\
\text { alto, el } 47.4 \% \\
\text { despersonalización alto y } \\
\text { un bajo nivel de realización } \\
\text { personal del } 22.7 \%, \text { de } \\
\text { igual manera se observó } \\
\text { que el miedo al contagio se } \\
\text { asoció con un alto nivel de } \\
\text { agotamiento y } \\
\text { despersonalización, de esta } \\
\text { manera es necesario } \\
\text { desarrollar políticas de } \\
\text { atención médica. }\end{array}$ \\
\hline 6 & Liu et al. (2020) & China & $\begin{array}{l}880 \text { médicos y } \\
\text { enfermeros }\end{array}$ & $\begin{array}{l}\text { Chinese } \\
\text { version of the } \\
\text { Maslach } \\
\text { Burnout } \\
\text { Inventory } \\
\text { (CMBI) }\end{array}$ & \begin{tabular}{|lr} 
Identificar el nivel \\
de burnout & y \\
descubrir & los \\
factores & de \\
asociación & \\
relacionados con el & brote de Covid-19
\end{tabular} & $\begin{array}{l}\text { Se ha identificado un alto } \\
\text { nivel de burnout en los } \\
\text { participantes, además las } \\
\text { dimensiones de } \\
\text { agotamiento emocional y la } \\
\text { despersonalización son } \\
\text { altos, juntamente con un } \\
\text { bajo nivel de realización } \\
\text { personal. Otro de los datos } \\
\text { importantes radica en que } \\
\text { los factores que } \\
\text { contribuyen a la presencia } \\
\text { del burnout son los turnos } \\
\text { nocturnos, juntamente con } \\
\text { la sintomatología, de } \\
\text { covid19. }\end{array}$ \\
\hline 7 & $\begin{array}{l}\text { Matsuo et al. } \\
(2020)\end{array}$ & Japón & $\begin{array}{l}312 \\
\text { participantes }\end{array}$ & $\begin{array}{l}\text { Japanese } \\
\text { version of the } \\
\text { Maslach } \\
\text { Burnout } \\
\text { Inventory- } \\
\text { General } \\
\text { Survey }\end{array}$ & $\begin{array}{lr}\text { Evaluar } & \text { la } \\
\text { prevalencia } & \text { del } \\
\text { síndrome } & \text { de } \\
\text { burnout entre } & \text { los } \\
\text { colaboradores } & \\
\text { sanitarios } & \text { de } \\
\text { primera } & \text { línea } \\
\text { durante } & \text { la } \\
\text { pandemia } & \text { de } \\
\text { COVID-19 } & \text { en } \\
\text { Japón } & \end{array}$ & $\begin{array}{l}\text { La prevalencia del Burnout } \\
\text { fue de } 31.4 \% \text { es decir } \\
\text { presente en } \\
\text { colaboradores de } 312 \text {, se } \\
\text { identificó además que el } \\
\text { agotamiento como tal está } \\
\text { más presente en mujeres, } \\
\text { siendo esto significativo en } \\
\text { comparación con los } \\
\text { hombres. De modo general } \\
\text { se infiere que más del } 40 \% \\
\text { de enfermeras, 30\% de } \\
\text { tecnólogos radiólogos y } \\
\text { farmacéuticos cumplían los } \\
\text { criterios para burnout. }\end{array}$ \\
\hline
\end{tabular}




\begin{tabular}{|c|c|c|c|c|c|c|}
\hline 8 & $\begin{array}{l}\text { Azoulay et } \\
\text { al.(2020) }\end{array}$ & - & $\begin{array}{l}1001 \\
\text { miembros del } \\
\text { ESICM }\end{array}$ & $\begin{array}{lr}\text { Inventario } & \text { de } \\
\text { Burnout } & \text { de } \\
\text { Maslach }(\mathrm{MBI})\end{array}$ & $\begin{array}{lr}\text { Conocer } & \text { la } \\
\text { prevalencia } & \text { del } \\
\text { síndrome } & \text { de } \\
\text { burnout entre } & \text { los } \\
\text { intensivistas } & \text { que } \\
\text { enfrentaron r el } & \text { el } \\
\text { brote de } & \text { COVID- } \\
19 & \end{array}$ & $\begin{array}{l}\text { Desde un enfoque } \\
\text { descriptivo se ha observado } \\
\text { que se trabajó en mayor } \\
\text { medida con intensivistas de } \\
\text { Europa Central (25\%), } \\
\text { Europa del Sur (23\%), } 12 \% \\
\text { de Reino Unido, 9\% de } \\
\text { América del Sur, } 8.1 \% \text { de } \\
\text { América del Norte, 5.3\% } \\
\text { de Europa del Este, } \\
\text { mientras que solo Oriente } \\
\text { Medio conformó el 5\%, el } \\
4.7 \% \text { América del Norte, } \\
\text { Asia por su parte de } 3.3 \% \\
2.7 \% \text { de India, Australia - } \\
\text { Nueva Zelanda el 1.3\%, } \\
\text { mientras que África el .6\%. } \\
\text { De estos participantes el } \\
51 \% \text { evidenció burnout } \\
\text { severo, sin embargo, los } \\
\text { niveles de agotamiento } \\
\text { tienen una variación } \\
\text { significativa según región, } \\
\text { finalmente se considera que } \\
\text { la pandemia ha tenido un } \\
\text { impacto significativo en los } \\
\text { especialistas de UCI, con } \\
\text { más del 50\% con } \\
\text { sintomatología severa, por } \\
\text { otro lado, los factores que } \\
\text { pueden desencadenar es el } \\
\text { desconocimiento y y } \\
\text { experiencia sobre la } \\
\text { enfermedad de la Covid-19. }\end{array}$ \\
\hline 9 & $\begin{array}{l}\text { Gómez-Galan et } \\
\text { al. (2020) }\end{array}$ & España & $\begin{array}{l}2182 \\
\text { participantes }\end{array}$ & $\begin{array}{l}\text { Maslach } \\
\text { Burnout } \\
\text { Inventory } \\
\text { (MBI) }\end{array}$ & $\begin{array}{l}\text { Determinar los } \\
\text { niveles de burnout } \\
\text { en profesionales de } \\
\text { tiendas de campaña }\end{array}$ & $\begin{array}{l}\text { De acuerdo con la variable } \\
\text { se ha identificado que se } \\
\text { presentan niveles altos a } \\
\text { nivel de sus subescalas del } \\
28.5 \% \text { es decir en } \\
\text { agotamiento emocional } \\
>50 \% \text {, despersonalización } \\
>55 \% \text { y la falta de } \\
\text { desarrollo personal } \\
\text { equivalente al } 46.3 \% \text {. } \\
\text { Finalmente, el trabajo en } \\
\text { situaciones como la de una } \\
\text { pandemia se traduce en una } \\
\text { enfermedad de carácter } \\
\text { ocupacional. }\end{array}$ \\
\hline
\end{tabular}




\begin{tabular}{|c|c|c|c|c|c|c|}
\hline 10 & $\begin{array}{l}\text { Abdelhafiz et al. } \\
(2020)\end{array}$ & Egipto & 220 médicos & $\begin{array}{l}\text { Maslach } \\
\text { Burnout } \\
\text { Inventory } \\
\text { (MBI) }\end{array}$ & $\begin{array}{l}\text { Evaluar la } \\
\text { frecuencia y los } \\
\text { factores de riesgo } \\
\text { asociados al SB en } \\
\text { médicos durante la } \\
\text { Pandemia. }\end{array}$ & $\begin{array}{l}\text { Se registró una prevalencia } \\
\text { del SB de } 36.36 \% \text { en los } \\
\text { médicos, en relación con } \\
\text { las características } \\
\text { sociodemográficas los de } \\
\text { menor edad tuvieron mayor } \\
\text { presencia del agotamiento } \\
\text { emocional } \\
\text { despersonalización } \\
\text { mientras que alcanzaron un } \\
\text { nivel de rendimiento } \\
\text { personal reducido, en tanto } \\
\text { resulta importante } \\
\text { desarrollo ayuda para los } \\
\text { médicos, como el apoyo } \\
\text { psicológico, las horas de } \\
\text { trabajo organizados, ajuste } \\
\text { salario y suministros de } \\
\text { protección personal como } \\
\text { medidas de bioseguridad. }\end{array}$ \\
\hline 11 & Tan et al. (2020) & Singapur & $\begin{array}{l}3075 \\
\text { trabajadores } \\
\text { de salud }\end{array}$ & $\begin{array}{l}\text { Oldenburg } \\
\text { Burnout } \\
\text { Inventory } \\
\text { (OLBI) }\end{array}$ & $\begin{array}{l}\text { Examinar } \\
\text { burnout y los } \\
\text { factores asociados } \\
\text { en trabajadores de } \\
\text { la salud en medio } \\
\text { de la pandemia } \\
\text { COVID-19 }\end{array}$ & $\begin{array}{l}\text { Se ha evidenciado niveles } \\
\text { de burnout considerable, } \\
\text { además el } 79.7 \% \text { y el } \\
75.3 \% \text { de los participantes } \\
\text { alcanzaron umbrales de } \\
\text { burnout en desconexión y } \\
\text { agotamiento, por otro lado, } \\
\text { se han considerado factores } \\
\text { como la duración de los } \\
\text { turnos y la reubicación para } \\
\text { la prevalencia de burnout } \\
\text { alto, finalmente el desgaste } \\
\text { se vio más afectada por las } \\
\text { tareas de alto riesgo por la } \\
\text { pandemia. }\end{array}$ \\
\hline 12 & $\begin{array}{l}\text { Dimitriu et al. } \\
(2020)\end{array}$ & Rumania & $\begin{array}{l}100 \text { residentes } \\
\text { médicos }\end{array}$ & $\begin{array}{l}\text { Maslach } \\
\text { Burnout } \\
\text { Inventory } \\
\text { (MBI) }\end{array}$ & $\begin{array}{lr}\text { Comparar las } \\
\text { frecuencias del } \\
\text { burnout en } \\
\text { médicos residentes } \\
\text { de primera línea y } \\
\text { los que trabajan en } \\
\text { salas normales }\end{array}$ & $\begin{array}{l}\text { De acuerdo a los datos se } \\
\text { ha observado dos grupos } \\
\text { que presentan } \\
\text { homogeneidad a nivel de } \\
\text { edad y sexo, sin embargo } \\
\text { de manera general se } \\
\text { encontró un nivel promedio } \\
\text { de SB (76\%), a nivel de } \\
\text { grupo se ha identificado } \\
\text { que el personal que está en } \\
\text { área no COVID registro un } \\
\text { SB del 88\% mientras que } \\
\text { los ubicados en primera } \\
\text { línea (emergencia, } \\
\text { radiología, unidad de } \\
\text { cuidado intensivo) solo } \\
\text { alcanzaron 66\%, sin } \\
\text { embargo lo de mayor } \\
\text { preocupación es que estos } \\
\text { índices sean con personal } \\
\text { que se encuentra en una } \\
\text { edad máximo de } 35 \text { años } \\
\text { que teóricamente se } \\
\text { deberían adaptar mejor a } \\
\text { los escenarios de pandemia. }\end{array}$ \\
\hline
\end{tabular}




\begin{tabular}{|c|c|c|c|c|c|c|}
\hline 13 & $\begin{array}{l}\text { Barello et al. } \\
(2020)\end{array}$ & Italia & $\begin{array}{l}1153 \\
\text { profesionales } \\
\text { sanitarios }\end{array}$ & $\begin{array}{l}\text { Maslach } \\
\text { Burnout } \\
\text { Inventory } \\
\text { (MBI) }\end{array}$ & $\begin{array}{lr}\text { Describir } & \text { los } \\
\text { niveles } & \text { de } \\
\text { agotamiento y } & \text { la } \\
\text { sintomatología } & \\
\text { física en } & \text { los } \\
\text { trabajadores } & \text { de } \\
\text { salud italianos } & \text { de } \\
\text { primera } & \text { línea } \\
\text { involucrados } & \text { en la } \\
\text { atención } & \text { de } \\
\text { pacientes } & \text { Covid-19 }\end{array}$ & 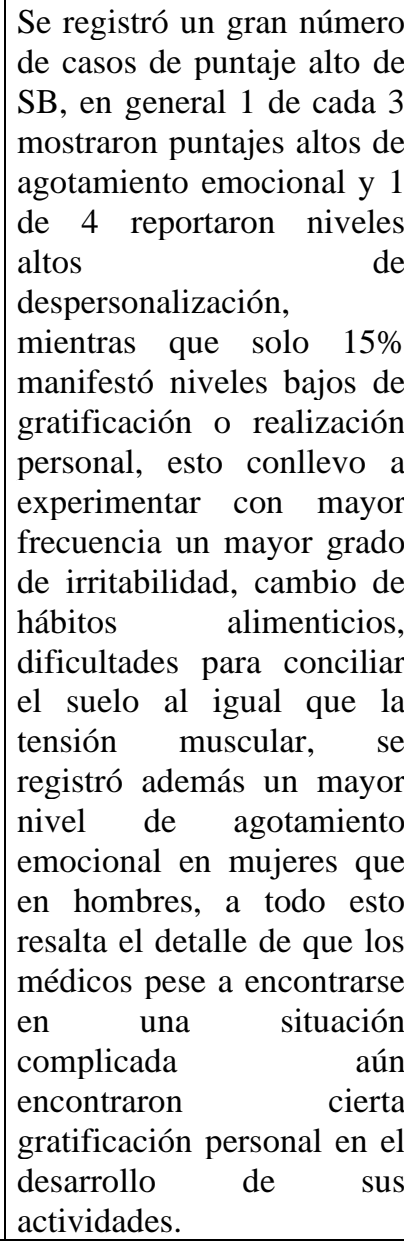 \\
\hline 14 & $\begin{array}{lll}\text { Giusti } & \text { et } & \text { al. } \\
(2020) & & \end{array}$ & Italia & \begin{tabular}{|l}
330 \\
profesionales \\
de la salud
\end{tabular} & $\begin{array}{l}\text { Maslach } \\
\text { Burnout } \\
\text { Inventory } \\
\text { (MBI) }\end{array}$ & \begin{tabular}{|lr} 
Evaluar & la \\
prevalencia & del \\
burnout y & las \\
condiciones & \\
psicopatológicas & \\
en los & los \\
profesionales de la & salud durante la \\
pandemia & de \\
Covid-19 &
\end{tabular} & $\begin{array}{l}\text { El 35.7\% de personal } \\
\text { presentó niveles de } \\
\text { agotamiento emocional } \\
\text { moderado y } 31.9 \% \text { severo, } \\
\text { el } 14 \% \text { tuvo niveles de } \\
\text { despersonalización } \\
\text { moderada y el } 34.3 \% \\
\text { presentó niveles severos de } \\
\text { realización personal, } \\
\text { finalmente, las condiciones } \\
\text { que tienden a intervenir } \\
\text { para llegar a niveles altos } \\
\text { se encuentran establecidos } \\
\text { por la edad, ocupación, } \\
\text { horario de trabajo y } \\
\text { contacto con pacientes } \\
\text { COVID-19 por el miedo a } \\
\text { contagiarse y a su familia. }\end{array}$ \\
\hline 15 & $\begin{array}{l}\text { Murat et al. } \\
(2020)\end{array}$ & Estambul & $\begin{array}{l}705 \\
\text { enfermeras }\end{array}$ & $\begin{array}{l}\text { Maslach } \\
\text { Burnout } \\
\text { Inventory } \\
\text { (MBI) }\end{array}$ & \begin{tabular}{|lr} 
Determinar & los \\
niveles de & estrés, \\
depresión & y \\
burnout de & las \\
enfermeras & de \\
primera & línea \\
durante & la \\
pandemia & de \\
COVID-19 &
\end{tabular} & $\begin{array}{l}\text { Se evidenció niveles altos } \\
\text { de estrés y burnout y } \\
\text { depresión moderada, se } \\
\text { demuestra además que los } \\
\text { niveles de afección } \\
\text { psicológica se registran } \\
\text { más en mujeres que } \\
\text { hombres. }\end{array}$ \\
\hline
\end{tabular}




\begin{tabular}{|c|c|c|c|c|c|c|}
\hline 16 & $\begin{array}{l}\text { Kholmogorova } \\
\text { et al. (2020) }\end{array}$ & Rusia & 102 médicos & $\begin{array}{l}\text { Maslach } \\
\text { Burnout } \\
\text { Inventory } \\
\text { (MBI) }\end{array}$ & $\begin{array}{lr}\text { Evaluar } & \text { la } \\
\text { presencia } & \text { de } \\
\text { burnout, depresión } \\
\text { y ansiedad } & \text { en } \\
\text { personal } & \text { médico } \\
\text { que } & \text { brinda } \\
\text { atención } & \text { a } \\
\text { pacientes } & \text { con } \\
\text { COVID-19 } & \end{array}$ & $\begin{array}{l}\text { El 35\% alcanzó un alto } \\
\text { nivel de agotamiento, } \\
\text { mientras que el } 8.3 \% \\
\text { presentó sintomatología de } \\
\text { ansiedad moderada y } \\
\text { severa, mientras que el } \\
6.9 \% \text { presentó } \\
\text { pensamientos suicidas, es } \\
\text { decir un nivel de depresión } \\
\text { relativamente bajo. La } \\
\text { atención a pacientes } \\
\text { COVID-19 contribuyen en } \\
\text { la sintomatología. }\end{array}$ \\
\hline 17 & $\begin{array}{l}\text { Roslan et al. } \\
(2021)(2021)\end{array}$ & Malasia & $\begin{array}{l}1126 \text { personal } \\
\text { médico }\end{array}$ & $\begin{array}{l}\text { Copenhagen } \\
\text { Burnout } \\
\text { Inventory } \\
\text { (CBI) }\end{array}$ & $\begin{array}{lr}\text { Examinar ra } & \text { la } \\
\text { prevalencia } & \text { del } \\
\text { agotamiento y } & \text { sus } \\
\text { factores que re } \\
\text { asocian en los } \\
\text { trabajadores de la } \\
\text { salud en Malasia }\end{array}$ & $\begin{array}{l}\text { Más del 50\% de los } \\
\text { colaboradores evaluados } \\
\text { presentaron agotamiento, } \\
\text { dentro de este escenario se } \\
\text { vio como factores } \\
\text { principales para la } \\
\text { prevalencia del SB la } \\
\text { participación directa en la } \\
\text { detección o tratamiento de } \\
\text { pacientes COVID-19, así } \\
\text { también el agotamiento se } \\
\text { fue influenciado por los } \\
\text { factores como carga } \\
\text { laboral, incertidumbre de la } \\
\text { pandemia, la relaciones } \\
\text { entre familia y trabajo. } \\
\end{array}$ \\
\hline 18 & $\begin{array}{l}\text { Lasalvia et al. } \\
\text { (Lasalvia et al., } \\
\text { 2021) }\end{array}$ & Italia & $\begin{array}{l}2195 \\
\text { trabajadores } \\
\text { médicos }\end{array}$ & $\begin{array}{l}\text { Maslach } \\
\text { Burnout } \\
\text { Inventory- } \\
\text { General } \\
\text { Survey (MBI- } \\
\text { GS) }\end{array}$ & $\begin{array}{l}\text { Determinar los } \\
\text { niveles de burnout } \\
\text { y los factores } \\
\text { asociados entre el } \\
\text { personal sanitario } \\
\text { que labora en un } \\
\text { hospital terciario } \\
\text { del noreste de } \\
\text { Italia durante la } \\
\text { pandemia r de } \\
\text { COVID-19 }\end{array}$ & $\begin{array}{l}\text { A nivel de dimensiones se } \\
\text { encontró que el el } \\
\text { agotamiento emocional es } \\
\text { alto en } 38.3 \% \text { la eficacia } \\
\text { profesional es bajo en } \\
46.5 \% \text { y un alto nivel de } \\
\text { cinismo (26.5\%), en ese } \\
\text { sentido el riesgo de } \\
\text { agotamiento aumentó de } \\
\text { acuerdo con el puesto, es } \\
\text { decir ser residente. }\end{array}$ \\
\hline 19 & $\begin{array}{l}\text { Sunjaya et al. } \\
(2020)\end{array}$ & Indonesia & $\begin{array}{l}544 \text { personal } \\
\text { de salud }\end{array}$ & $\begin{array}{l}\text { Inventario de } \\
\text { Burnout (BOI) }\end{array}$ & $\begin{array}{l}\text { Explorar la } \\
\text { presencia de la } \\
\text { sintomatología de } \\
\text { depresión ansiedad } \\
\text { y burnout en } \\
\text { personal de salud } \\
\text { un más después del } \\
\text { brote de Covid-19 }\end{array}$ & $\begin{array}{l}\text { El nivel de agotamiento } \\
\text { presentes en el personal } \\
\text { médico es considerable } \\
>26 \% \text { estos a su vez se ven } \\
\text { influenciados por el } \\
\text { contacto y trata de } \\
\text { pacientes con COVID-19 } \\
\text { estableciendo una clara } \\
\text { diferencia con aquellos } \\
\text { médicos que no tratan a } \\
\text { estos pacientes. }\end{array}$ \\
\hline
\end{tabular}

De los artículos evaluados se observó una mayor prevalencia originaria de Italia (3) a diferencia del resto provenientes de otras partes del mundo (Tabla 1), las evaluaciones a los artículos se han trabajado con un tipo aplicado, de diseño descriptivo observacional transversal (17). solo se consideró un estudio correlacional y uno longitudinal.

Se han trabajado en su totalidad con profesionales de la salud como médicos, enfermeros, residentes, psicólogos, terapistas, entre otros con un total de muestra de 19624 sujetos, siendo el mínimo 100, además más del 90\% de los instrumentos aplicados fueron el Inventario de Burnout de 
Maslach, en ese sentido dentro de los principales objetivos se plantearon identificar los niveles de burnout en profesionales de la salud durante la pandemia. Cabe precisar que también se buscaron identificar los factores de riesgo asociados al mismo y se han efectuado comparaciones de acuerdo con las áreas de la institución.

El burnout registrado en los profesionales de la salud tuvo un nivel mínimo del 15\% llegando hasta el $95 \%$ de estos, la mayor prevalencia se registró en profesionales de sexo femenino con edades inferiores a los 35 años. En relación con sus componentes, los niveles de agotamiento emocional han demostrado estar en un nivel alto entre el $30 \%$ y $70 \%$ en profesionales de la salud que atienden de manera directa a pacientes con diagnóstico y sintomatología de la COVID-19, es decir, aquellos que se encuentran en la primera línea de atención (Abdelhafiz et al., 2020; Barello et al., 2020; Lasalvia et al., 2021). Dentro de estas características las mujeres tienden a estar más predispuestas para el desarrollo de esta afección, a consecuencia del grado de involucramiento (Duarte et al., 2020; Elhadi et al., 2020); similares resultados se evidencian en cuanto a la despersonalización que presenta altos niveles relacionados con el tiempo laboral, área y el grado de experiencia (Gómez-Galán et al., 2020; Liu et al., 2020); en cuanto al tercer componente, la realización personal fue moderada, además, no se registraron evidencias de diferencia estadística entre las demás áreas que están involucrados en el proceso de atención a pacientes (Giusti et al., 2020).

Dentro de los factores que se han observado de mayor presencia fueron el mantener contacto con paciente COVID-19, de igual manera la ubicación en áreas de alto riesgo como UCI en más del $50 \%$, además que los turnos juntamente con la carga laboral son factores relevantes para la presencia de sintomatología. Por otro lado, el miedo al contagio, la incertidumbre, el desconocimiento de la enfermedad, el estrés y las relaciones entre el personal han estado presentes.

\subsection{Discusión}

Del análisis de los datos se perciben que la prevalencia de burnout en todo el mundo, si bien han presentado sus propias características, sin embargo, el nivel de estos es alarmante, no solo por sus consecuencias sino también por todos los factores psicosociales que están involucradas, SilvaGomes y Silva-Gomes (2021) plantea como principales la ansiedad, depresión, preocupación y altos niveles de estrés. Por su parte Giorgi et al. (2020) adiciona la prevalencia en personal de primera línea con mayor predisposición; siendo estos con mayor tendencia a presentar problemas psiquiátricos (Galli et al., 2020; Navinés et al., 2021).

El registro de altos índices de burnout revela el efecto que ha tenido la COVID-19 sobre la salud y estado emocional del personal médico, más aún centrados en personal médico de sexo femenino, guardando así una estrecha relación con los datos obtenidos por Al et al. (2020) que llegaron a concluir que la COVID-19 ha tenido un efecto negativo en el personal médico de Arabia Saudita, además de manifestar predictores para la presencia de una afección psicológica como la edad, sexo, exposición a eventos traumáticos, entre otros; datos similares al obtenido en el estudio refuerzan aún más estas características, donde es preciso referenciar a los factores de angustia e incertidumbre, miedo al contagio y los niveles elevados de estrés por periodos prolongados. De igual manera se observan factores como la carga excesiva del trabajo con pacientes COVID-19, contribuyendo como carga psicológica para la presencia de agotamiento (Silva-Gomes \& SilvaGomes, 2021; Yakut et al., 2020). Por otro lado, a nivel de áreas estos han presentado variaciones según la complejidad, riesgo e interacción con pacientes, como manifiestan Ruíz-Fernández et al. (2020) el personal médico en área específica de COVID-19 presentan niveles más elevados de afección psicológica.

Resulta importante orientar al apoyo psicológico, además de desarrollar medidas para ajustar los horarios y evitar que diversos factores, externos como internos afecten el desarrollo de las actividades laborales, de igual manera desarrollar diversas estrategias que contribuyan en la 
reducción del Burnout, como lo manifestado por De Moura et al. (2020) quienes plantean que el establecimiento de relación de alta calidad con los supervisores y el personal médico en primera línea para mitigar el impacto del agotamiento, así también la COVID-19 como tal representa un riesgo para el bienestar psicológico y mental de los colaboradores, razón por la que necesita una intervención de alta calidad de acorde con las especificaciones del centro laboral o institución de salud pública (Khalafallah et al., 2020; Raudenská et al., 2020; Salazar et al., 2020), estos tienen que ser desarrollados de acuerdo a la evaluación de impacto en la salud mental (Lozano-Vargas, 2021).

Los factores que predisponen la aparición de burnout son múltiples, sin embargo, se registro en estudios que el agotamiento emocional y la despersonalización son los que mayor impacto negativo tienen, estos han sido evaluados desde mucho tiempo, por lo que se sugiere que el estrés, los roles, conflictos y eventos estresantes predisponen su aparición (Sonnentag, 2001); en relación a este último, se ha visto reforzado por la severidad del problema en salud a consecuencia de la pandemia; no obstante, es preciso manifestar, que la COVID-19 no ha sido el único factor que propició la presencia del burnout en los profesionales de la salud de primera línea, siendo así el desencadenante por la acumulación de diversos estresores a través del tiempo, como la sobrecarga de jornadas laborales además de la no valoración de la salud mental en el centro laboral (Mancebo, 2020).

De acuerdo con los hallazgos evidenciados, se ha reforzado en mayor medida el modelo tripartito de Maslach et al. (2001), que mediante la utilización del instrumento direcciona a su mejor entendimiento y aplicabilidad dentro de los diversos escenarios o circunstancias, como el caso de pandemia actual, permitiendo así medir la intensidad en que se desarrolla, como bien es sabido existen más de mil síntomas que pueden ser característicos del burnout, no obstante este modelo lo agrupa en sus tres componentes (Gil-Monte \& Peiró, 1999; Olivares, 2017) como respuesta a los estresores dentro de la institución donde desarrolla sus actividades.

De las limitaciones presentadas en el estudio, se consideraron el número de artículos orientados al tema en específico, sobre todo de acceso abierto, de igual manera el estudio solo abarcó el año 2020 y los dos primeros meses del 2021, donde las evaluaciones de burnout estuvieron en fases evaluativas, por otro lado llama la atención, que a nivel mundial no se ha prestado la debida atención de los que sus consecuencias del burnout implica en la salud de los profesionales al igual que su repercusión en el paciente, más aún en América del sur, donde la evaluación se han presentado a niveles mínimos de $8.1 \%$ de evaluación (Azoulay et al., 2020); finalmente, resulta importante que a partir de los datos se puedan ampliar así el número de muestra y sobre todo el tipo de participantes, como personal docente, servidores públicos, entre otro tipo de personal de atención directa al cliente de empresas públicas o privadas.

\section{CONCLUSIÓN}

El nivel de burnout es alto en el personal médico el mismo que se presentó desde el 15\% al 95\%. Se han identificado índices mayores en mujeres, los datos también manifiestan que se la sintomatología ha estado más presente en el personal médico más joven, para la presencia de burnout, el contacto con pacientes COVID-19, los horarios laborales, la sobrecarga laboral, el estrés y el desconocimiento o tratamiento de la COVID-19 han sido un factor primordial. El agotamiento emocional junto con la despersonalización y la realización personal, han afectado en mayor medida de manera independiente en el desarrollo de sus funciones, además, la evaluación se ha efectuado casi en su totalidad por el IBM, por agrupar las diversas sintomatologías en sus tres componentes. Finalmente resulta prudente que se desarrolle estrategias de atención psicológica en las unidades de atención primaria a pacientes, garantizando así el bienestar psicológico de los profesionales de la salud. A partir de los resultados del estudio es importante que se explore en futuras investigaciones la comparación de los índices de burnout antes de la pandemia y durante del mismo a fin de 
descubrir el efecto que ha tenido la crisis actual, finalmente, desarrollar estudios bibliométricos involucrando artículos con muchas de diversas áreas.

\section{REFERENCIAS}

Abdelhafiz, A. S., Ali, A., Ziady, H. H., Maaly, A. M., Alorabi, M., \& Sultan, E. A. (2020). Prevalence, Associated Factors, and Consequences of Burnout Among Egyptian Physicians During COVID-19 Pandemic. Frontiers in Public Health, 8(590190), 1-9. https://doi.org/10.3389/fpubh.2020.590190

Al, E., Mosli, M., \& Alameel, T. (2020). The psychological impact of COVID-19 pandemic on physicians in Saudi Arabia: A cross-sectional study. Saudi Journal of Gastroenterology, 26(5), 249-255. https://doi.org/10.4103/sjg.SJG_174_20

Amanullah, S., \& Shankar, R. R. (2020). The Impact of COVID-19 on Physician Burnout Globally: A Review. Healthcare, 8(4), 421. https://doi.org/10.3390/HEALTHCARE8040421

Ato, M., Juan, L., \& Benavente, A. (2013). Un sistema de clasificación de los diseños de investigación en psicología. Anales de Psicologia, 29(3), 1038-1059. https://doi.org/10.6018/analesps.29.3.178511

Azoulay, E., De Waele, J., Ferrer, R., Staudinger, T., Borkowska, M., Povoa, P., Iliopoulou, K., Artigas, A., Schaller, S. J., Hari, M. S., Pellegrini, M., Darmon, M., Kesecioglu, J., \& Cecconi, M. (2020). Symptoms of burnout in intensive care unit specialists facing the COVID-19 outbreak. Annals of Intensive Care, 10(1), 1-8. https://doi.org/10.1186/s13613-020-00722-3

Barello, S., Palamenghi, L., \& Graffigna, G. (2020). Burnout and somatic symptoms among frontline healthcare professionals at the peak of the Italian COVID-19 pandemic. Psychiatry Research, 290(113129). https://doi.org/10.1016/j.psychres.2020.113129

Burki, T. K. (2020). Burnout among cancer professionals during COVID-19. The Lancet Oncology, 21(11), 1402. https://doi.org/10.1016/S1470-2045(20)30584-2

Carlin, M., \& Garcés De Los Fayos, E. J. (2010). El síndrome de burnout: Evolución histórica desde el contexto laboral al ámbito deportivo. Anales de psicología, 26, 169-180. https://www.redalyc.org/pdf/167/16713758020.pdf

Çelmeçe, N., \& Menekay, M. (2020). The Effect of Stress, Anxiety and Burnout Levels of Healthcare Professionals Caring for COVID-19 Patients on Their Quality of Life. Frontiers in Psychology, 11(597624), 1-7. https://doi.org/10.3389/fpsyg.2020.597624

Cherniss, C. (1993). Role of professional self-efficacy in the etiology and amelioration of burnout. En W. B. Schaufeli, C. Maslach, \& T. Marek (Eds.), Taylor \& Francis. Taylor \& Francis. https://psycnet.apa.org/record/1993-97794008

De Moura, E., Furtado, L., \& Sobral, F. (2020). The Burnout Epidemic During The Covid-19 Pandemic: The Role of LMX in alleviating Physician’s Burnout. Revista de Administração de Empresas, 60(6), 426-436. https://doi.org/10.1590/S0034-759020200606

Dimitriu, M. C. T., Pantea-Stoian, A., Smaranda, A. C., Nica, A. A., Carap, A. C., Constantin, V. D., Davitoiu, A. M., Cirstoveanu, C., Bacalbasa, N., Bratu, O. G., Jacota-Alexe, F., Badiu, C. D., Smarandache, C. G., \& Socea, B. (2020). Burnout syndrome in Romanian medical residents in time of the COVID-19 pandemic. Medical Hypotheses, 144(109972). https://doi.org/10.1016/j.mehy.2020.109972

Duarte, I., Teixeira, A., Castro, L., Marina, S., Ribeiro, C., Jácome, C., Martins, V., Ribeiro-Vaz, I., Pinheiro, H. C., Silva, A. R., Ricou, M., Sousa, B., Alves, C., Oliveira, A., Silva, P., Nunes, R., \& Serrão, C. (2020). Burnout among Portuguese healthcare workers during the COVID-19 pandemic. BMC Public Health, 20, 2-10. https://doi.org/10.1186/512889-020-09980-7

El-Hage, W., Hingray, C., Lemogne, C., Yrondi, A., Brunault, P., Bienvenu, T., Etain, B., Paquet, C., Gohier, B., Bennabi, D., Birmes, P., Sauvaget, A., Fakra, E., Prieto, N., Bulteau, S., Vidailhet, P., Camus, V., Leboyer, M., Krebs, M. O., \& Aouizerate, B. (2020). Health professionals facing the coronavirus disease 2019 (COVID-19) pandemic: What are the mental health risks? Encephale, 46(3), S73-S80. https://doi.org/10.1016/j.encep.2020.04.008

Elhadi, M., Msherghi, A., Elgzairi, M., Alhashimi, A., Bouhuwaish, A., Biala, M., Abuelmeda, S., Khel, S., Khaled, A., Alsoufi, A., Elmabrouk, A., Alshiteewi, F. Bin, Hamed, T. Ben, Alhadi, B., Alhaddad, S., Elhadi, A., \& Zaid, A. (2020). Burnout Syndrome Among Hospital Healthcare Workers During the COVID-19 Pandemic and Civil War: A Cross-Sectional Study. Frontiers in Psychiatry, 11(579563), 1-11. https://doi.org/10.3389/fpsyt.2020.579563 
Galli, F., Pozzi, G., Ruggiero, F., Mameli, F., Cavicchioli, M., Barbieri, S., Canevini, M. P., Priori, A., Pravettoni, G., Sani, G., \& Ferrucci, R. (2020). A Systematic Review and Provisional Metanalysis on Psychopathologic Burden on Health Care Workers of Coronavirus Outbreaks. Frontiers in Psychiatry, 11(568664). https://doi.org/10.3389/FPSYT.2020.568664

Gil-Monte, P. R., \& Peiró, J. M. (1999). Perspectivas teóricas y modelos interpretativos para el estudio del síndrome de quemarse por el trabajo Title: A review of models and theoretical perspectives to study of professional burnout. Anales de psicología, 15(2), 261-268. https://www.um.es/analesps/v15/v15_2pdf/12v98_05Llag2.PDF

Giorgi, G., Lecca, L. I., Alessio, F., Finstad, G. L., Bondanini, G., Lulli, L. G., Arcangeli, G., \& Mucci, N. (2020). COVID-19-Related Mental Health Effects in the Workplace: A Narrative Review. International Journal of Environmental Research and Public Health, 17(21), 1-22. https://doi.org/10.3390/IJERPH17217857

Giusti, E. M., Pedroli, E., Aniello, G. E. D., Badiale, C. S., Pietrabissa, G., Manna, C., Badiale, M. S., \& Riva, G. (2020). The Psychological Impact of the COVID-19 Outbreak on Health Professionals : A Cross-Sectional Study. Frontiers in Psychology, 11, 1-9. https://doi.org/10.3389/fpsyg.2020.01684

Gómez-Galán, J., Lázaro-Pérez, C., Martínez-López, J. Á., \& Fernández-Martínez, M. D. M. (2020). Burnout in spanish security forces during the covid-19 pandemic. International Journal of Environmental Research and Public Health, 17(23), 1-15. https://doi.org/10.3390/ijerph17238790

Hoseinabadi, T. S., Kakhki, S., Teimori, G., \& Nayyeri, S. (2020). Burnout and its influencing factors between frontline nurses and nurses from other wards during the outbreak of Coronavirus Disease -COVID-19- in Iran. Nursing Research and Education, 38(2), e03.

Juárez-García, A. (2020). Síndrome de burnout en personal de salud durante la pandemia COVID-19: un semáforo naranja en la salud mental. Salud UIS, 52(1), 8. https://doi.org/10.18273/revsal.v52n4-2020010

Khalafallah, A. M., Lam, S., Gami, A., Dornbos, D. L., Sivakumar, W., Johnson, J. N., \& Mukherjee, D. (2020). A national survey on the impact of the COVID-19 pandemic upon burnout and career satisfaction among neurosurgery residents. Journal of Clinical Neuroscience, 80, 137-142. https://doi.org/10.1016/j.jocn.2020.08.012

Kholmogorova, A. B., Petrikov, S. S., Suroyegina, A. Y., Mikita, O. Y., Rakhmanina, A. A., \& Roy, A. P. (2020). Burnout and its Factors in Healthcare Specialists Involved in Providing Care for Patients with Covid-19 at Different Stages of the Pandemic. Kholmogorova, 9(3), 1-18. https://doi.org/10.23934/2223-9022-2020-9-3-321337 Burnout

Lasalvia, A., Amaddeo, F., Porru, S., Carta, A., Tardivo, S., Bovo, C., Ruggeri, M., \& Bonetto, C. (2021). Levels of burn-out among healthcare workers during the COVID-19 pandemic and their associated factors: a cross-sectional study in a tertiary hospital of a highly burdened area of north-east Italy. BMJ Open, 11(1), e045127. https://doi.org/10.1136/BMJOPEN-2020-045127

Liu, X., Chen, J., Wang, D., Li, X., Wang, E., Jin, Y., Ma, Y., Yu, C., Luo, C., Zhang, L., Liu, C., Zhou, Y., Yang, L., Song, J., Bai, T., \& Hou, X. (2020). COVID-19 Outbreak Can Change the Job Burnout in Health Care Professionals. Frontiers in Psychiatry, 11(563781), 1-9. https://doi.org/10.3389/fpsyt.2020.563781

Lozano-Vargas, A. (2021). El síndrome de burnout en los profesionales de salud en la pandemia por la COVID-19. Revista Neurosiquiatría, 84(1), 1-2. https://doi.org/10.20453/rnp.v84i1.3930

Mancebo, S. (2020, julio 6). Médicos españoles, burnout tras la pandemia COVID-19. Efe:salud. https://www.efesalud.com/burnout-medicos-espanoles-quemados-pandemia-covid-19/

Maslach, C., Schaufeli, W. B., \& Leiter, M. P. (2001). Job Burnout. Annual Review of Psychology, 52, $397-422$. https://doi.org/10.1146/ANNUREV.PSYCH.52.1.397

Matsuo, T., Kobayashi, D., Taki, F., Sakamoto, F., Uehara, Y., Mori, N., \& Fukui, T. (2020). Prevalence of Health Care Worker Burnout During the Coronavirus Disease 2019 (COVID-19) Pandemic in Japan. JAMA network open, 3(8), e2017271. https://doi.org/10.1001/jamanetworkopen.2020.17271

Moher, D., Liberati, A., Tetzlaff, J., Altman, D. G., \& Group, T. P. (2009). Preferred Reporting Items for Systematic Reviews and Meta-Analyses: The PRISMA Statement. PLOS Medicine, 6(7), e1000097. https://doi.org/10.1371/JOURNAL.PMED.1000097

Montero, I., \& León, O. (2002). Clasificación y descripción de las metodologías de. Revista Internacional de Psicología Clínica y de la Salud, 2(3), 503-508. http://www.aepc.es/ijchp/articulos_pdf/ijchp-53.pdf

Murat, M., Köse, S., \& Savaşer, S. (2020). Determination of stress , depression and burnout levels of front-line nurses during the COVID-19 pandemic. International Journal of Mental Health Nursing, 30(2). 
https://doi.org/10.1111/inm.12818

Navinés, R., Olivé, V., Fonseca, F., \& Martín-Santos, R. (2021). Estrés laboral y burnout en los médicos residentes, antes y durante la pandemia por COVID-19: una puesta al día. Medicina Clinica, 157(3), 140. https://doi.org/10.1016/J.MEDCLI.2021.04.003

Olivares, V. (2017). Laudatio: Dra. Christina Maslach, Comprendiendo el Burnout. Ciencia \& trabajo, 19(58), 59-63. https://scielo.conicyt.cl/pdf/cyt/v19n58/0718-2449-cyt-19-58-00059.pdf

Otzen, T., \& Manterola, C. (2017). Técnicas de Muestreo sobre una Población a Estudio. International Journal of Morphology, 35(1), 227-232. https://doi.org/10.4067/S0717-95022017000100037

Raudenská, J., Steinerová, V., Javůrková, A., Urits, I., Kaye, A. D., Viswanath, O., \& Varrassi, G. (2020). Occupational burnout syndrome and post-traumatic stress among healthcare professionals during the novel coronavirus disease 2019 (COVID-19) pandemic. Best Practice and Research: Clinical Anaesthesiology, 34(3), 553-560. https://doi.org/10.1016/j.bpa.2020.07.008

Reed, T. (1981). Burn-Out: Stages of Disillusionment in the Helping Professions. By Jerry Edelwhich and Archie Brodsky. New York: Human Sciences Press, 1980. NASSP Bulletin, 65(445), 106-106. https://doi.org/10.1177/019263658106544520

Ricci-Cabello, I., Meneses-Echavez, J. F., Serrano-Ripoll, M. J., Pastor, G., Castro, A., Ruiz-Pérez, I., Zamanillo, R., \& Gonçalves-Bradley, D. (2020). Impact of viral epidemic outbreaks on mental health of healthcare workers: a rapid systematic review. BMJ, 1-92. https://doi.org/10.1101/2020.04.02.20048892

Rodríguez, B. O., \& Sánchez, T. L. (2020). The psychosocial impact of COVID-19 on health care workers. International Braz J Urol, 46(Suppl 1), 195-200. https://doi.org/10.1590/S1677-5538.IBJU.2020.S124

Roslan, N. S., Saiful, M., Yusoff, B., Asrenee, A. R., \& Morgan, K. (2021). Burnout Prevalence and Its Associated Factors among Malaysian Healthcare Workers during COVID-19 Pandemic : An Embedded Mixed-Method Study. Healthcare Article, 9(90), 1-20. https://doi.org/10.3390/healthcare9010090

Ruiz-Fernández, M. D., Ramos-Pichardo, J. D., Ibáñez-Masero, O., Cabrera-Troya, J., Carmona-Rega, M. I., \& OrtegaGalán, Á. M. (2020). Compassion fatigue, burnout, compassion satisfaction and perceived stress in healthcare professionals during the COVID-19 health crisis in Spain. Journal of Clinical Nursing, 29(21-22), 4321-4330. https://doi.org/10.1111/jocn.15469

Salazar, G., Vaquerizo-Serrano, J., Catalan, A., Arango, C., Moreno, C., Ferre, F., Shin, J. Il, Sullivan, S., Brondino, N., Solmi, M., \& Fusar-Poli, P. (2020). Impact of coronavirus syndromes on physical and mental health of health care workers: Systematic review and meta-analysis. Journal of Affective Disorders, 275, 48-57. https://doi.org/10.1016/J.JAD.2020.06.022

Sanghera, J., Pattani, N., Hashmi, Y., Varley, K. F., Cheruvu, M. S., Bradley, A., \& Burke, J. R. (2020). The impact of SARS-CoV-2 on the mental health of healthcare workers in a hospital setting-A Systematic Review. Journal of Occupational Health, 62(1), e12175. https://doi.org/10.1002/1348-9585.12175

Seow, J. W., Lin, S., Khor, Y. P., Hey, D. H., \& Kagda, F. H. (2020). Stress and Strain in an Orthopaedic Department on the Frontlines during the COVID-19 Pandemic: An Analysis of Burnout and the Factors Influencing It. Annals of the Academy of Medicine, Singapore, 49(10), 814-816. https://doi.org/10.47102/annals-acadmedsg.2020289

Sharifi, M., Asadi-Pooya, A. A., \& Mousavi-Roknabadi, R. S. (2020). Burnout among Healthcare Providers of COVID19; a Systematic Review of Epidemiology and Recommendations. Archives of Academic Emergency Medicine, 9(1), 1-17. https://doi.org/10.22037/aaem.v9i1.1004

Silva-Gomes, R. N., \& Silva-Gomes, V. T. (2021). Pandemia de la COVID-19: síndrome de Burnout en profesionales sanitarios que trabajan en hospitales de campaña en Brasil. Enfermería Clínica, 31(2), 128-129. https://doi.org/10.1016/J.ENFCLI.2020.10.011

Sonnentag, S. (2001). Well-being and Burnout in the Workplace, Psychology of. International Encyclopedia of the Social \& Behavioral Sciences, 16445-16447. https://doi.org/10.1016/B0-08-043076-7/01396-6

Stuijfzand, S., Deforges, C., Sandoz, V., Sajin, C.-T., Jaques, C., Elmers, J., \& Horsch, A. (2020). Psychological impact of an epidemic/pandemic on the mental health of healthcare professionals: a rapid review. BMC Public Health 2020 20:1, 20(1), 1-18. https://doi.org/10.1186/S12889-020-09322-Z

Sunjaya, D. K., Herawati, D. M. D., \& Siregar, A. Y. (2020). Depressive , Anxiety , and Burnout Symptoms on Health Care Personnel at a Month After COVID-19 Outbreak in Indonesia : A Documentary Research Using Rasch Model Analysis. Research Square, 21, 1-13. https://doi.org/10.1186/s12889-021-10299-6

Tan, B. Y. Q., Kanneganti, A., Lim, L. J. H., Tan, M., Chua, Y. X., Tan, L., Sia, C. H., Denning, M., Goh, E. T., 
Purkayastha, S., Kinross, J., Sim, K., Chan, Y. H., \& Ooi, S. B. S. (2020). Burnout and Associated Factors Among Health Care Workers in Singapore During the COVID-19 Pandemic. Journal of the American Medical Directors Association, 21(12), 1751-1758.e5. https://doi.org/10.1016/j.jamda.2020.09.035

Veléz, R. R., Echavez, J. M., \& Flórez, M. E. (2013). Una propuesta metodológica para la conducción de revisiones sistemáticas de la literatura en la investigación biomédica Methodology in conducting a systematic review of biomedical research. undefined, 1, 61-73. http://132.248.161.133:8080/jspui/handle/123456789/5467

Vinueza, A. F., Aldaz, N. R., Mera, C. M., Pino, D. P., Tapia, E. C., \& Vinueza, M. F. (2020). Síndrome de Burnout en médicos/as y enfermeros/as ecuatorianos durante la pandemia deCOVID-19. Psychiatria Damubina, 1-21. https://doi.org/10.1590/SciELOPreprints.708

Visser, A. (2020). Burnout is now an 'occupational phenomenon': Changes to how people work amid the coronavirus pandemic are bearing down on their psychological well-being, which has implications for employers. finWeek, 44-45. https://www.news24.com/fin24/finweek/burnout-is-now-an-occupational-phenomenon-20201001

Yakut, E., Kuru, Ö., \& Güngör, Y. (2020). Sağlık Personelinin Covid-19 Korkusu ile Tükenmişliği Arasındaki İlişkide Aşırı İş Yükü ve Algılanan Sosyal Desteğin Etkisinin Yapısal Eşitlik Modeliyle Belirlenmesi. Ekev Akademi Dergisi, 83(Yaz), 241-261. https://doi.org/10.17753/Ekev1621 